\title{
BMJ Open High-flow nasal cannula oxygen therapy alone or with non-invasive ventilation during the weaning period after extubation in ICU: the prospective randomised controlled HIGH- WEAN protocol
}

Arnaud W Thille, ${ }^{1,2}$ Grégoire Muller, ${ }^{3}$ Arnaud Gacouin, ${ }^{4}$ Rémi Coudroy, ${ }^{1,2}$ Alexandre Demoule, ${ }^{5}$ Romain Sonneville, ${ }^{6}$ François Beloncle, ${ }^{7}$ Christophe Girault ${ }^{8}$ Laurence Dangers, ${ }^{9}$ Alexandre Lautrette, ${ }^{10}$ Séverin Cabasson, ${ }^{11}$ Anahita Rouzé, ${ }^{12}$ Emmanuel Vivier, ${ }^{13}$ Anthony Le Meur, ${ }^{14}$ Jean-Damien Ricard, ${ }^{15}$ Keyvan Razazi, ${ }^{16}$ Guillaume Barberet, ${ }^{17}$ Christine Lebert, ${ }^{18}$ Stephan Ehrmann, ${ }^{19}$ Walter Picard, ${ }^{20}$ Jeremy Bourenne, ${ }^{21}$ Gael Pradel, ${ }^{22}$ Pierre Bailly, ${ }^{23}$ Nicolas Terzi, ${ }^{24}$ Matthieu Buscot, ${ }^{25}$ Guillaume Lacave, ${ }^{26}$ Pierre-Eric Danin, ${ }^{27}$ Hodanou Nanadoumgar, ${ }^{28}$ Aude Gibelin, ${ }^{29}$ Lassane Zanre, ${ }^{30}$ Nicolas Deye, ${ }^{31}$ Stéphanie Ragot, ${ }^{2}$ Jean-Pierre Frat, ${ }^{1,2}$ For the REVA research network

To cite: Thille AW, Muller G, Gacouin A, et al. High-flow nasal cannula oxygen therapy alone or with non-invasive ventilation during the weaning period after extubation in ICU: the prospective randomised controlled HIGHWEAN protocol. BMJ Open 2018;8:e23772. doi:10.1136/ bmjopen-2018-023772

- Prepublication history for this paper is available online. To view these files, please visit the journal online (http://dx.doi. org/10.1136/bmjopen-2018023772).

Received 25 April 2018 Revised 12 July 2018 Accepted 20 July 2018

Check for updates

(c) Author(s) (or their employer(s)) 2018. Re-use permitted under CC BY-NC. No commercial re-use. See rights and permissions. Published by BMJ.

For numbered affiliations see end of article.

Correspondence to Professor Arnaud W Thille; aw.thille@gmail.com

\section{ABSTRACT}

Introduction Recent practice guidelines suggest applying non-invasive ventilation (NIV) to prevent postextubation respiratory failure in patients at high risk of extubation failure in intensive care unit (ICU). However, such prophylactic NIV has been only a conditional recommendation given the low certainty of evidence. Likewise, high-flow nasal cannula (HFNC) oxygen therapy has been shown to reduce reintubation rates as compared with standard oxygen and to be as efficient as NIV in patients at high risk. Whereas HFNC may be considered as an optimal therapy during the postextubation period, HFNC associated with NIV could be an additional means of preventing postextubation respiratory failure. We are hypothesising that treatment associating NIV with HFNC between NIV sessions may be more effective than HFNC alone and may reduce the reintubation rate in patients at high risk.

Methods and analysis This study is an investigatorinitiated, multicentre randomised controlled trial comparing HFNC alone or with NIV sessions during the postextubation period in patients at high risk of extubation failure in the ICU. Six hundred patients will be randomised with a 1:1 ratio in two groups according to the strategy of oxygenation after extubation. The primary outcome is the reintubation rate within the 7 days following planned extubation. Secondary outcomes include the number of patients who meet the criteria for moderate/severe respiratory failure, ICU length of stay and mortality up to day 90 .

Ethics and dissemination The study has been approved by the ethics committee and patients will be included after informed consent. The results will be submitted for publication in peer-reviewed journals. Trial registration number NCT03121482.
Strengths and limitations of this study

- High-flow nasal cannula (HFNC) oxygen therapy had never previously been used as a reference therapy. When administered to a control group, this treatment seemed highly innovative and in agreement with the recent literature. Likewise, the ventilatory strategy used in the interventional group associating non-invasive ventilation (NIV) and HFNC had never previously been assessed.

- This study will be the largest randomised controlled trial never conducted on the use of NIV during the postextubation period and may help to establish strong recommendations on weaning strategy with a high level of evidence.

- A large population of patients considered to be at high risk for reintubation will be included. Patients older than 65 years or those with an underlying chronic cardiac or respiratory disease are easy to identify in clinical practice and represent nearly half of the patients who are extubated in the intensive care unit.

- The individual study assignments of the patients will not be masked. Given the characteristics of the two strategies under evaluation, a double-blind trial is not possible.

\section{INTRODUCTION}

Background and rationale

The day of extubation is a critical time during an intensive care unit (ICU) stay because in case of extubation failure, mortality can 
Table 1 Main randomised controlled trials having assessed the use of prophylactic NIV to prevent postextubation respiratory failure in ICU

\section{Randomised controlled trials}

No of centres/inclusion criteria

Nava et $a l^{3}$
3 centres: NIV $(n=48)$ vs $\mathrm{O}_{2}(n=49)$
Patients considered at high risk for reintubation
Ferrer et $\mathrm{al}^{5}$
2 centres: NIV $(\mathrm{n}=79)$ vs $\mathrm{O}_{2}(\mathrm{n}=83)$
Age $>65$, APACHE II>12 or intubation for cardiac heart
failure

Ferrer et $a l^{6}$

$\mathrm{PCO}_{2}>45 \mathrm{~mm} \mathrm{Hg}$ at the end of the spontaneous breathing trial
3 centres: NIV $(n=54)$ vs $\mathrm{O}_{2}(n=52)$

\section{Main results: NIV versus standard oxygen $\left(\mathrm{O}_{2}\right)$}

Reintubation: $n=4$ ( $8 \%)$ vs $n=12(24 \%), p=0.027^{*}$ In-ICU mortality: $n=3$ (6\%) vs $n=9(18 \%), p=0.064$

Respiratory failure after extubation: $n=13$ (16\%) vs $n=27$ (33\%), $\mathrm{p}=0.029^{*}$

Reintubation: $n=9$ (11\%) vs $n=18(22 \%), p=0.12$

Nosocomial Infections: $18(23 \%)$ vs 27 (33\%), $p=N S$

In-ICU mortality: NIV $n=2$ (3\%) vs. $n=12(14 \%), p=0.015$

Respiratory failure after extubation: $n=8$ (15\%) vs $n=25$ (48\%), $\mathrm{p}<0.0001^{*}$

Reintubation: $n=6(11 \%)$ vs $n=10(19 \%), p=0.37$

Pneumonias: 3 (6\%) vs 9 (17\%), $p=0.12$

In-ICU mortality: $n=3$ (6\%) vs $n=4(8 \%), p=0.71$

Mortality at day $90: n=6(11 \%)$ vs $n=16(31 \%), p=0.024$

Khilnani et $a l^{26}$
1 single centre: NIV $(n=20)$ vs $\mathrm{O}_{2}(n=20)$

Reintubation: $n=5$ (25\%) vs $n=3(15 \%), p=0.44^{*}$

Length of ICU stay: $18.3 \pm 7.9 \mathrm{j}$ vs $16.1 \pm 6.3, \mathrm{p}=0.34$

\section{Su et $\left.a\right|^{27}$ \\ 3 centres: NIV $(n=202)$ vs $\mathrm{O}_{2}(n=204)$ \\ Intubation $\geq 48$ hours}

Ornico et $a l^{4}$

1 single centre: NIV $(n=20)$ vs $\mathrm{O}_{2}(n=18)$

Intubation $\geq 3$ days for respiratory failure

Vargas et al ${ }^{28}$
6 centres: NIV $(n=71)$ vs $\mathrm{O}_{2}(n=72)$
Patients with chronic lung disorders

Vargas et $a l^{28}$

Patients with chronic lung disorders
Extubation failure: $n=30$ (15\%) vs $n=27(13 \%), p=0.62 *$

Reintubation: $n=21(10 \%)$ vs $n=16(8 \%), p=0.37$

In-ICU mortality: $n=3(1.5 \%)$ vs $n=2(1 \%), p=0.64$

Reintubation: $n=1$ (5\%) vs $n=7$ (39\%), $p=0.016$ *

In-hospital mortality: $0 \%$ vs $n=4(22 \%), p=0.041$

Respiratory failure after extubation: $n=6(8 \%)$ vs $n=20(28 \%)$, $\mathrm{p}=0.002^{*}$

Reintubation: $n=6(8 \%)$ vs $n=13(18 \%), p=0.09$

In-ICU mortality: $n=2(3 \%)$ vs $n=6(8 \%), p=0.28$

Mortality at day $90: n=7(10 \%)$ vs $n=11(15 \%), p=0.33$

*Main end point.

APACHE, Acute Physiology and Chronic Health Evaluation; COPD, chronic obstructive pulmonary disease; ICU, intensive care unit; NIV, noninvasive ventilation; $\mathrm{PCO}_{2}$, partial pressure of carbon dioxide.

reach $30 \%-50 \% .^{12}$ The overall rate of extubation failure is around $10 \%-15 \%$, but it may exceed $20 \%$ in patients at high risk. ${ }^{2}$ Several studies suggest that prophylactic non-invasive ventilation (NIV) applied within the first 24-48 hours after extubation could reduce the risk of respiratory failure in patients at high risk table $1 .^{3-8}$ Recent European/American clinical practice guidelines have suggested that NIV be used to prevent postextubation respiratory failure in patients at high risk. ${ }^{9}$ These guidelines specified that for most of these studies, patients at high risk of extubation failure included those $>65$ years or with underlying cardiac or respiratory disease. $^{35-8}$ In a before-after study assessing the implementation of a specific prophylactic NIV programme, we observed a significant reduction in the reintubation rate when prophylactic NIV was systematically applied after extubation in this population at high risk. ${ }^{8}$ A meta-analysis of randomised controlled trials also suggests that prophylactic NIV may decrease reintubation rates in this population. ${ }^{10}$ However, that has not been demonstrated in large randomised controlled trials and only two small randomised controlled studies have shown that NIV decreased reintubation rates as compared with standard oxygen. ${ }^{34}$ Therefore, use of prophylactic NIV was a conditional recommendation in recent international guidelines, given the low certainty of evidence. ${ }^{9}$

Up until now, the majority of patients have been treated with standard oxygen after extubation. ${ }^{11}$ High-flow nasal cannula oxygen therapy (HFNC) is an alternative device for oxygenation that improves gas exchange and reduces the work of breathing. ${ }^{12-14}$ Two randomised controlled trials have shown a significant reduction in the reintubation rate for patients treated with HFNC as compared with standard oxygen. ${ }^{15} 16$ In another large-scale randomised controlled trial, HFNC was equivalent to NIV in patients at high risk of extubation failure. ${ }^{17}$ Therefore, HFNC may be considered as the reference therapy during the postextubation period. In order to further improve gas exchange and the work of breathing, HFNC may be associated with NIV.

\section{Objectives}

We aim to conduct a prospective multicentre randomised controlled trial comparing HFNC alone or with NIV 
sessions during the postextubation period in patients at high risk of extubation failure in the ICU, with the hypothesis that treatment associating HFNC with NIV may reduce the reintubation rate as compared with HFNC alone.

\section{Primary objective}

To compare the reintubation rate within the 7 days following planned extubation between HFNC alone and HFNC with NIV in patients at high risk of extubation failure in the ICU.

\section{Secondary objectives}

To compare the number of patients who meet the criteria for moderate/severe respiratory failure within the 7 days following extubation, and the rates of reintubation at 48 hours, 72 hours and up to ICU discharge.

To compare the number of ventilator-free days within the 14 days following extubation, length of stay in ICU and in the hospital and, mortality in ICU, in hospital, at day 28 and up to day 90 between the two groups.

\section{Trial design}

The HIGH-FLOW for WEANING (HIGH-WEAN) study is an investigator-initiated, prospective, multicentre, randomised, control, open trial comparing a strategy of oxygenation during the postextubation period with HFNC alone or with NIV in patients at high risk of extubation failure in the ICU. Patients will be randomly assigned to the NIV group or HFNC group, with a 1:1 ratio.

\section{METHODS: PARTICIPANTS, INTERVENTIONS AND OUTCOMES Study setting}

The HIGH-WEAN study is taking place in 30 ICUs in France.

\section{Eligibility criteria \\ Inclusion criteria}

Adult patients intubated more than 24 hours in ICU and at high risk of extubation failure will be eligible if considered ready for extubation by the physician in charge and after success of a spontaneous breathing trial performed according to the international conference consensus on weaning (figure 1). ${ }^{18}$

Patients will be considered at high risk of extubation failure according to the following criteria ${ }^{19}$ : patients older than 65 years, or those having any underlying chronic cardiac or lung disease. Underlying chronic cardiac diseases include left ventricular dysfunction whatever the cause defined by left ventricular ejection fraction $\leq 45 \%$, history of cardiogenic pulmonary oedema, documented ischaemic heart disease or permanent atrial fibrillation. Chronic lung diseases include the existence of any underlying chronic obstructive pulmonary disease (COPD), obesity hypoventilation syndrome (OHS) or restrictive pulmonary disease. The underlying lung disease will be either documented or highly suspected by the physician in a patient intubated for acute hypercapnic respiratory failure and having (1) a history of smoking with intrinsic positive end-expiratory pressure (PEEP) during mechanical ventilation and/or emphysema on chest X-ray or scanner suggesting underlying COPD, (2) obesity body mass index $\left(>30 \mathrm{~kg} / \mathrm{m}^{2}\right)$ with alveolar hypoventilation arterial carbon dioxide tension $\left(\mathrm{PaCO}_{2}>45 \mathrm{~mm} \mathrm{Hg}\right)$ suggesting OHS or (3) rib cage deformation suggesting restrictive pulmonary disease.

\section{Exclusion criteria}

Patients fulfilling one of the following criteria will not be included: patients admitted for traumatic brain injury or with any underlying chronic neuromuscular disease (myopathy or myasthenia gravis), patients with NIV or continuous positive airway pressure at home, or with a contraindication to NIV, and patients with do-not-reintubate order at time of extubation or unplanned extubation (accidental or self extubation).

\section{Intervention}

Oxygenation strategy during the postextubation period

Patients eligible for inclusion will be randomised at time of decision of planned extubation and assigned to one of the two following groups: (1) The patients assigned to control group will receive continuously HFNC alone and (2) The patients assigned to interventional group will receive NIV during at least 12 hours a day with HFNC between NIV sessions.

As NIV may be more effective in hypercapnic patients, blood gas will be systematically assessed at the end of the spontaneous breathing trial prior to extubation in order to stratify the randomisation for $\mathrm{PaCO}_{2}$ level and to include the same number of hypercapnic patients $\left(\mathrm{PaCO}_{2}>45 \mathrm{~mm} \mathrm{Hg}\right)$ in the two groups.

\section{Control group: HFNC alone}

Immediately after planned extubation, the patients assigned to the control group will be continuously treated by HFNC for at least 48 hours with a flow of $50 \mathrm{~L} / \mathrm{min}$ and fractional inspired oxygen $\left(\mathrm{FiO}_{2}\right)$ adjusted to obtain adequate oxygenation (Pulse Oximetry $\left(\mathrm{SpO}_{2}\right) \geq 92 \%$ ). To provide sufficient humidification the temperature of the heated humidifier will be set as during invasive mechanical ventilation, that is, at $37^{\circ} \mathrm{C}$.

\section{Interventional group: HFNC and NIV}

NIV will be immediately initiated after planned extubation with a first session of at least 4 hours and then by sessions of at least 2 hours for a minimal duration of at least 12 hours a day during the 48 hours following extubation. Continuous application of NIV will be promoted throughout the entire night period (between 22:00 and 6:00 hours). NIV will be carried out with a ventilator dedicated for NIV (ICU ventilator with NIV mode or NIV ventilator) in pressure-support ventilation with a minimal PS level of $5 \mathrm{~cm} \mathrm{H}_{2} \mathrm{O}$ targeting a tidal volume around $6-8 \mathrm{~mL} / \mathrm{kg}$, a PEEP level between 5 and 10 $\mathrm{cm} \mathrm{H}_{2} \mathrm{O}$ and $\mathrm{FiO}_{2}$ adjusted to obtain adequate oxygenation $\left(\mathrm{SpO}_{2} \geq 92 \%\right)$. Between NIV sessions, HFNC 
Screening of all extubations in ICU

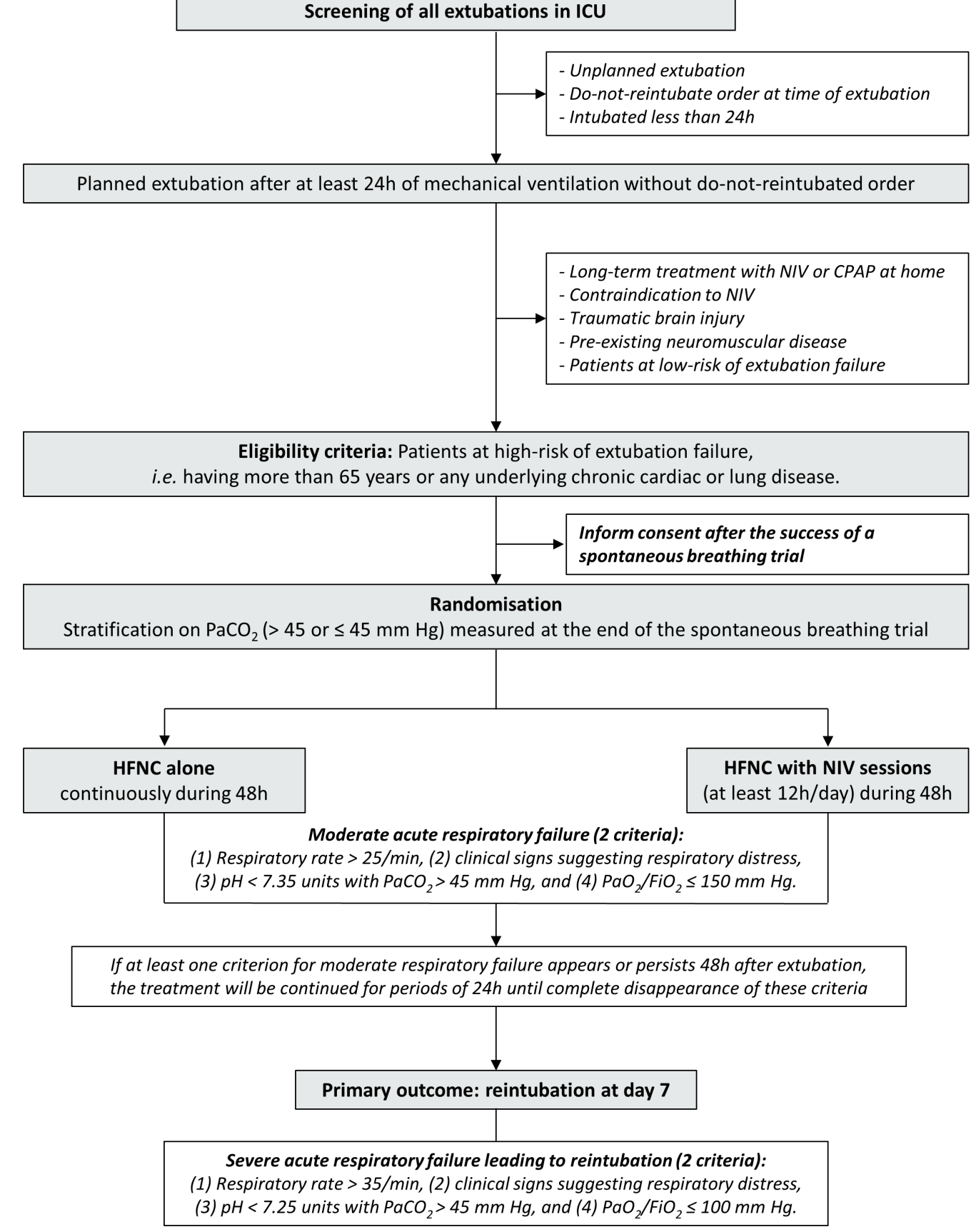

Figure 1 Flow chart of the patients and study design. Patients extubated after at least 24 hours of mechanical ventilation and without do-not-reintubate order will be eligible if they are considered at high risk of extubation failure, that is, more than 65 years old or with underlying chronic cardiac or respiratory disease. Patients will be randomised and treated either with high-flow nasal cannula (HFNC) oxygen therapy alone or with sessions of non-invasive ventilation (NIV) with at least 12 hours a day of NIV. Forty-eight hours after planned extubation, treatment will be stopped or continued according to patient respiratory status. CPAP, continuous positive airway pressure; $\mathrm{FiO}_{2}$, fractional inspired oxygen; ICU, intensive care unit; $\mathrm{PaO}$, arterial oxygen tension; $\mathrm{PACO}_{2}$, arterial carbon dioxide tension.

will be delivered as in the control group with a flow of $50 \mathrm{~L} / \mathrm{min}$ and $\mathrm{a} \mathrm{FiO}_{2}$ to achieve adequate oxygenation $\left(\mathrm{SpO}_{2} \geq 92 \%\right)$.

In both groups, therapeutic NIV used to treat postextubation respiratory failure will be discouraged given that it has no proven benefit ${ }^{20}$ and can even increase the risk of death by delaying reintubation. ${ }^{21}$

\section{Duration of treatment}

In the two groups, patients will be treated for a minimal duration of 48 hours. After that, continuation of the treatment will be decided according to the patient respiratory status (figure 1).

If none of the criteria for moderate respiratory failure are present 48 hours after extubation (see criteria below), 
treatment will be stopped and switched to standard oxygen therapy. If at least one criterion for moderate respiratory failure appears or persists 48 hours after extubation, the allocated treatment will be continued for periods of 24 hours until complete disappearance of these respiratory criteria.

Criteria for moderate respiratory failure include the following: (1) Respiratory rate $>25 / \mathrm{min}$ persistent at least 2 hours, (2) Clinical signs suggesting respiratory distress with increase in the work of breathing and/or respiratory fatigue including activation of accessory respiratory muscles, (3) Respiratory acidosis defined as $\mathrm{pH}<7.35$ units and $\mathrm{PaCO}_{2}>45 \mathrm{~mm} \mathrm{Hg}$, (4) Hypoxaemia defined as a need for $\mathrm{FiO}_{2} \geq 50 \%$ to maintain $\mathrm{SpO}_{2} \geq 92 \%$ or arterial oxygen tension $\left(\mathrm{PaO}_{2}\right) / \mathrm{FiO}_{2} \leq 150 \mathrm{~mm} \mathrm{Hg}$.

An episode of moderate acute respiratory failure during the postextubation period will be defined by the presence of at least two criteria for moderate respiratory failure.

\section{Outcomes}

Primary outcome

The primary outcome is reintubation within the 7 days following planned extubation.

Patients will be reintubated only if one of the following criteria occurs:

1. Severe acute respiratory failure defined by the presence of at least two criteria for severe respiratory failure among the following: (1) Respiratory rate $>35 / \mathrm{min}$, (2) Clinical signs suggesting respiratory distress with increase in the work of breathing and/or respiratory fatigue including activation of accessory respiratory muscles, (3) Respiratory acidosis defined as $\mathrm{pH}<7.25$ units and $\mathrm{PaCO}_{2}>45 \mathrm{~mm} \mathrm{Hg}$, (4) Hypoxaemia defined as a need for $\mathrm{FiO}_{2} \geq 80 \%$ to maintain $\mathrm{SpO}_{2} \geq 92 \%$ or $\mathrm{PaO}_{2} / \mathrm{FiO}_{2} \leq 100 \mathrm{~mm} \mathrm{Hg}$.

2. Haemodynamic failure defined as systolic arterial pressure $<90 \mathrm{~mm} \mathrm{Hg}$ or mean arterial pressure $<65 \mathrm{~mm} \mathrm{Hg}$ with a need for vasopressors.

3. Neurological failure: altered consciousness (Glasgow $<12$ ) or agitation.

4. Cardiac or respiratory arrest.

\section{Secondary outcomes}

Secondary outcome variables include the following:

1. Reintubation at 48 hours, 72 hours and up until ICU discharge.

2. An episode of moderate acute respiratory failure within the 7 days following extubation.

3. An episode of severe acute respiratory failure within the 7 days following extubation.

4. Number of ventilator-free days within the 14 days following extubation.

5. Length of stays in ICU and in hospital.

6. Mortality in ICU, in hospital, at day 28 and at day 90.

\section{Sample size}

We determined that enrolment of 590 patients would provide a power of $80 \%$ to show an absolute difference of $8 \%$ in the rate of reintubation between the control group using HFNC alone (rate of reintubation estimated to $18 \%$ ) as compared with the interventional group using HFNC and NIV (rate of reintubation estimated to 10\%) at a two-sided alpha level of 0.05 . As NIV may be more effective in hypercapnic patients, stratification will be performed in order to include the same number of hypercapnic patients $\left(\mathrm{PaCO}_{2}>45 \mathrm{~mm} \mathrm{Hg}\right)$ in the two groups.

\section{Expected rate of reintubation in the two groups}

The expected rates of reintubation in the two groups are based on the recent literature using HFNC in the postextubation period ${ }^{15-17}$ and on our preliminary studies assessing NIV in patients at high risk. ${ }^{8} 1922$

In our preliminary studies, the reintubation rate in patients at high risk treated with NIV was $15 \%$ within the 7 days following extubation. Although no study to date has evaluated a ventilatory strategy combining NIV and HFNC, we can expect an additionally decreased reintubation rate (around 10\%) in the interventional group.

Several studies have assessed HFNC alone during the postextubation period. ${ }^{15-17}$ The rates of reintubation reached $19 \%$ in the subset of patients at high risk. ${ }^{17}$ Although these rates could be underestimated (first, because the rate of reintubation was assessed within the first 48 or 72 hours following extubation and not at day 7 as in our study, and second because hypercapnic patients considered at high risk for reintubation were excluded), we expect a rate of reintubation at day 7 of around $15 \%-20 \%$ in the control group treated by HFNC alone.

\section{Recruitment}

The initial duration of patient enrolment expected is 2 years, starting in April 2017.

- End of 2015: national grant award.

- 2016: approval by an independent ethics committee.

- 2017: inclusion of patients.

- 2018-2019: end of inclusions, monitoring of participating centres and queries to investigators; overseen by the steering committee at the REVA Network meetings every 4 months; blind review to determine protocol violation, to define intention-to-treat and per-protocol analysis populations; new queries to investigators, cleaning and closure of the database.

- 2019: data analysis, writing of the manuscript and submission for publication.

\section{METHODS: ASSIGNMENT OF INTERVENTION, DATA COLLECTION, MANAGEMENT AND ANALYSIS}

Allocation and sequence intervention

A computer-generated randomisation is performed with stratification according to centre and $\mathrm{PaCO}_{2} \leq 45$ or $>45 \mathrm{~mm} \mathrm{Hg}$ measured at the end of the spontaneous breathing trial in a 1:1 ratio, using a centralised web-based management system (Clinfile). After randomisation, the strategy assigned to the patient (HFNC alone or with NIV) will be initiated immediately after extubation. 


\begin{tabular}{|c|c|c|c|c|c|c|}
\hline $\begin{array}{l}\text { Screening of spontaneous } \\
\text { breathing trials (SBT) }\end{array}$ & \begin{tabular}{c|} 
After a \\
successful SBT
\end{tabular} & $\begin{array}{r}\text { One hour after } \\
\text { extubation (H1) }\end{array}$ & $\begin{array}{l}\text { At } \mathrm{H6}, \mathrm{H} 12, \\
\mathrm{H} 24 \text {, and } \mathrm{H} 48\end{array}$ & \begin{tabular}{|l} 
From day \\
3 to day 7
\end{tabular} & $\begin{array}{l}\text { At day } 14 \text { and } \\
\text { ICU discharge }\end{array}$ & $\begin{array}{l}\text { At day } 28 \text { and day } \\
90 \text { (by phone) }\end{array}$ \\
\hline $\begin{array}{l}\text { Inclusion and non-inclusion } \\
\text { criteria }\end{array}$ & $x$ & & & & & \\
\hline $\begin{array}{l}\text { Inform consent from the patient } \\
\text { or relatives }\end{array}$ & $x$ & $x$ & $x$ & $x$ & $\mathrm{x}$ & $x$ \\
\hline Randomisation & $\mathrm{x}$ & & & & & \\
\hline $\begin{array}{l}\text { Reason for intubation and risk } \\
\text { factor for extubation failure }\end{array}$ & $x$ & & & & & \\
\hline $\begin{array}{l}\text { Demographic data and severity } \\
\text { scores }\end{array}$ & $x$ & & & & & \\
\hline Weaning difficulty & $\mathrm{x}$ & & & & & \\
\hline $\begin{array}{l}\text { Characteristics of the SBT } \\
\text { procedure }\end{array}$ & $x$ & & & & & \\
\hline $\begin{array}{l}\text { Clinical parameters, ventilatory } \\
\text { settings and blood gases }\end{array}$ & $\begin{array}{l}\text { At the end of } \\
\text { SBT }\end{array}$ & $x$ & $x$ & $x$ & & \\
\hline $\begin{array}{l}\text { Criteria for respiratory failure } \\
\text { and reintubation }\end{array}$ & & $x$ & $x$ & $x$ & & \\
\hline $\begin{array}{l}\text { Duration of NIV sessions and of } \\
\text { HFNC }\end{array}$ & & $x$ & $x$ & $x$ & $x$ & \\
\hline Status and length of stay & & $\mathrm{x}$ & $x$ & $x$ & $X$ & $x$ \\
\hline
\end{tabular}

Figure 2 Flow chart of the study showing timing collection of different variables. ICU, intensive care unit; HFNC, high-flow nasal cannula; NIV, non-invasive ventilation.

\section{Data collection and management}

Data will be collected on an Case Report Form (e-CRF) by a trained investigator or research assistant at each centre (figure 2). At time of inclusion, the following data on weaning procedure will be collected: type of spontaneous breathing trial performed before extubation (T-tube or pressure-support trial), vital parameters at the end of the spontaneous breathing trial and classification according to weaning difficulty. ${ }^{18}$ We will also collect qualitative assessment of cough strength and amount of secretions, and the use of steroids before extubation.

Ventilatory settings (gas flow and $\mathrm{FiO}_{2}$ using HFNC, pressure-support, $\mathrm{PEEP}, \mathrm{FiO}_{2}$ and expiratory tidal volume with NIV), and blood gas will be collected 1 hour after extubation and then at H6, between $\mathrm{H} 12$ and H24, and between $\mathrm{H} 24$ and H48. During the first 48 hours after extubation, we will collect the number and duration of NIV sessions and HFNC, criteria for moderate/severe acute respiratory failure and need for reintubation. All these parameters will be collected each day from day 3 to ICU discharge. Informed consent, intubation or tracheotomy, ventilator-free days at day 14 and length of ICU stay will be collected at ICU discharge while death will be collected by phone at day 90 .

\section{Statistical methods}

All the analyses will be performed by the study statistician according to a predefined statistical analysis plan and using statistical software (SAS, V.9.3; SAS Institute). A two-tailed $p<0.05$ will be considered as indicating statistical significance.

\section{Descriptive analysis of patient groups at baseline}

The analysis will be performed on an intention-to-treat basis after validation by a blind review committee of the inclusion and exclusion criteria for each patient. Wrongly included subjects as well as those lost to follow-up will be described. Deviations from the protocol will be described and analysed on a case-by-case basis.

Analysis pertaining to the main criteria of evaluation

Kaplan-Meier curves will be plotted to assess time from enrolment to reintubation and will be compared by means of the log-rank test at day 7 . The variables associated with reintubation with a $\mathrm{p}<0.20$ will be assessed by means of a Cox proportional hazard regression analysis applying a backward-selection procedure. The final model will include variables significantly associated with reintubation with a $\mathrm{p}<0.05$ and will be expressed using adjusted relative risk and HR with $95 \%$ CI. The percentages of patients having needed reintubation within the 7 days following planned extubation will be compared between the two groups by means of the $\chi^{2}$ test. The analysis will subsequently be completed by multivariate logistic regression after testing for interactions.

Analysis pertaining to the secondary criteria of evaluation

Reintubation rates at the various predefined times and moderate or severe acute respiratory failure rates will be compared between the two groups according to the same statistical methodology as the main outcome. Number of ventilator-free days and lengths of stay be compared between the two treatment groups by means of the Student's t-test. Regarding mortality criteria (at day 28 and at day 90), Kaplan-Meier curves will be plotted to assess the time from enrolment to death and will be compared between the two treatment groups by means of the log-rank test. A Cox proportional hazard regression analysis will be performed using a backward-manual procedure. 


\section{Predetermined subgroup analysis}

Randomisation will be stratified according to the $\mathrm{PaCO}_{2}$ value before extubation in order to obtain the same proportion of hypercapnic patients defined as a $\mathrm{PaCO}_{2}>45 \mathrm{~mm}$ $\mathrm{Hg}$ at the end of the spontaneous breathing trial. A subgroup analysis will consequently be performed for the main and secondary criteria of evaluation in hypercapnic patients with $\mathrm{PaCO}_{2}>45 \mathrm{~mm} \mathrm{Hg}$ before extubation and in non-hypercapnic patients, as well. Prior to adjustment, an interaction test will be carried out to detect heterogeneity of treatment effect between hypercapnic and non-hypercapnic patients. If the interaction test is positive, results will be given in two different subgroups.

We will also perform a subgroup analysis according to the existence of any underlying cardiac or lung disease, age, severity scores, type of spontaneous breathing trial, clinical parameters at the end of the spontaneous breathing trial, cough assessment, amount of secretions, use of steroids and weaning difficulty (simple, difficult or prolonged). As the duration of NIV may have an impact on outcome, we will also perform a subgroup analysis among patients having actually received at least 12 hours of NIV during the first 24 hours (dose recommended by the study protocol).

\section{Data monitoring}

An investigator at each centre will be responsible for daily patient screening, enrolling patients in the study, ensuring adherence to the protocol and completing the e-CRF. Research assistants will regularly monitor all the centres on site to check adherence to the protocol and the accuracy of the data recorded.

\section{ETHICS AND DISSEMINATION}

\section{Consent or assent}

The patient will be included after having provided a written informed consent to the investigator according to the decision of the central ethics committee. If the patient is not able to understand the information given, he/she can be included if the same procedure is completed with a next of kin. After the patient's recovery, he/she will be asked if he/she agrees to continue the trial.

\section{Confidentiality}

Data will be handled according to French law. All original records will be archived at trial sites for 15 years. The clean database file will be anonymised and kept for 15 years.

\section{Declaration of interest}

The HIGH-WEAN study is an investigator-initiated trial supported by the French Ministry of Health with funds obtained in 2015 from a national hospital clinical research programme (Programme Hospitalier de Recherche Clinique National 2015). The European research network REVA has endorsed the study project. The study is promoted by the University Hospital of
Poitiers. The firm Fisher \& Paykel Healthcare provides high-flow oxygen therapy equipment and face masks for NIV to all the participating centres but has no other involvement in the study.

\section{Access to data}

All investigators will have access to the final data set. Participant-level data sets will be made accessible on a controlled access basis.

\section{Dissemination policy}

Findings will be published in peer-reviewed journals and presented at local, national and international meetings and conferences to publicise and explain the research to clinicians, commissioners and service users.

\section{Patient and public involvement}

Patients and public were not involved in the study

\section{DISCUSSION}

Several studies have suggested that prophylactic NIV could reduce the risk of postextubation respiratory failure in ICU patients at high risk of extubation failure (table 1) ${ }^{3-8}$ NIV was started immediately after extubation and applied either continuously (at least 18 hours/day) during the first 24 hours $^{4}$ or by sessions of 1-2 hours for a total duration of at least 8 hours a day during the first 48 hours. ${ }^{348}$ Only three of these studies (two randomised controlled trials and one before-after study), all performed in single centre and including a small patient sample, have observed that reintubation rates were lower with NIV than with standard oxygen. ${ }^{348}$ The HIGH-WEAN study will be the largest randomised controlled trial ever conducted on the use of NIV during the postextubation period and may help to establish strong recommendations on weaning strategy with a high level of evidence.

Whereas previous studies have included a high proportion of hypercapnic patients, ${ }^{3-7}$ we decided to include a larger population of patients at high risk including patients older than 65 years or with an underlying chronic cardiac or respiratory disease. In a preliminary study, we found that prophylactic NIV systematically applied for 24 hours or more according to respiratory status reduced the reintubation rate in this population, from $28 \%$ to $15 \%$ $(\mathrm{p}=0.03)^{8}$ and enabled us to calculate the sample size of the present study. However, as NIV may be more effective in case of hypercapnia, we decided to stratify according to this variable at time of randomisation in order to have the same number of hypercapnic patients in the two groups and to plan subgroup analysis.

Although the international consensus conference on weaning defined extubation success as absence of ventilatory support during the first 48 hours after extubation, ${ }^{18}$ several studies have used a more prolonged time interval to assess extubation failure and reintubation. ${ }^{22-25}$ As prophylactic NIV may delay reintubation, the time interval needed to assess extubation failure 
should probably be longer than 48 hours in order to avoid underestimating extubation failure rates. We therefore decided to consider extubation failure in case of reintubation within the 7 days following planned extubation.

In our previous study, NIV was prolonged beyond the first 24 hours in more than $20 \%$ of the cases because of patients' respiratory status. ${ }^{8}$ Therefore, whatever the group of randomisation, all patients will receive HFNC alone or NIV interspaced with HFNC during at least 48 hours while this strategy may be continued beyond the first 48 hours in the absence of complete recovery.

The usual treatment after planned extubation includes standard oxygen alone through a facemask or nasal cannula. However, it has recently been shown that the use of HFNC after planned extubation decreased the rate of reintubation as compared with standard oxygen. ${ }^{15} 16$ In another study, the rate of reintubation was similar in high-risk patients treated with HFNC alone and in those treated with prophylactic NIV interspaced by standard oxygen. ${ }^{17}$ Therefore, HFNC may be an alternative to standard oxygen during the postextubation period. According to clinical practice in participating centres, the use of standard oxygen alone would have been considered as a suboptimal strategy for patients at high risk. The clinician must offer at any time the best possible treatment for the patient. If a clinician is convinced of the superiority of one treatment over another, then he has no reason to propose a randomised study comparing these two treatments. To promote equipoise and facilitate inclusions in different centres, we decided to use HFNC as ventilatory support in the control group.

In conclusion, the HIGH-WEAN trial is an investigator-initiated pragmatic randomised controlled trial empowered to test the hypothesis that NIV with HFNC may decrease reintubation rates after planned extubation of patients at high risk in ICU in comparison with HFNC alone. This study presents several innovative aspects. First, HFNC had never previously been used as a reference therapy although this treatment seems highly innovative and is in agreement with the recent literature. Likewise, the ventilatory strategy used in the interventional group associating NIV and HFNC had never previously been assessed.

\section{Author affiliations}

${ }^{1}$ Department of Réanimation Médicale, CHU de Poitiers, Poitiers, France

${ }^{2}$ Université de Poitiers, INSERM CIC 1402 ALIVE, Poitiers, France

${ }^{3}$ Médecine Intensive Réanimation, Groupe Hospitalier Régional d'Orléans, Orléans,

France

${ }^{4}$ Service des Maladies Infectieuses et Réanimation Médicale, CHU de Rennes, Hôpital Ponchaillou, Rennes, France

${ }^{5}$ Service de Pneumologie et Réanimation Médicale du Département R3S, AP-HP, INSERM, UMRS1158 Neurophysiologie Respiratoire Expérimentale et Clinique,

Sorbonne Université, Groupe Hospitalier Pitié-Salpêtrière Charles Foix, Paris, France ${ }^{6}$ Hôpital Bichat - Claude Bernard, Médecine Intensive Réanimation, AP-HP, Université Paris Diderot, Paris, France

${ }^{7}$ Département de Médecine Intensive - Réanimation, Université d'Angers, CHU d'Angers, Angers, France
${ }^{8}$ Département de Réanimation Médicale, Normandie Université, UNIROUEN, EA3830-GRHV, Institute for Research and Innovation in Biomedicine (IRIB), CHU de Rouen, Hôpital Charles Nicolle, Rouen, France

${ }^{9}$ Service de Réanimation Polyvalente, CHU Félix Guyon, Saint Denis de la Réunion, France

${ }^{10}$ Service de Réanimation Médicale, CHU de Clermont-Ferrand, Hôpital Gabriel Montpied, Clermont-Ferrand, France

${ }^{11}$ Service de Réanimation, Centre hospitalier de la Rochelle, La Rochelle, NouvelleAquitaine, France

${ }^{12}$ Centre de Réanimation, Université de Lille, CHU de Lille, Lille, France

${ }^{13}$ Reanimation Polyvalente, Hôpital Saint Joseph Saint Luc, Lyon, France

${ }^{14}$ Médecine Intensive Réanimation, CHU de Nantes, Nantes, France

${ }^{15}$ Réanimation Médico-Chirurgicale, AP-HP, INSERM, Université Paris Diderot, UMR IAME 1137, Sorbonne Paris Cité, Hopital Louis-Mourier, Colombes, France

${ }^{16}$ Service de Réanimation Médicale DHU A-TVB, AP-HP, Hopitaux Universitaires

Henri Mondor, Creteil, Île-de-France, France

${ }^{17}$ Service de Réanimation Médicale, Groupe Hospitalier Régional Mulhouse Sud Alsace, Site Emile Muller, Mulhouse, France

${ }^{18}$ Service de Médecine Intensive et Réanimation, Centre Hospitalier Départemental de Vendée, La Roche-sur-Yon, France

${ }^{19} \mathrm{CHU}$ de Tours, Médecin Intensive Réanimation, CIC 1415, CRICS-TriggerSEP, Centre d'étude des pathologies respiratoires, INSERM U1100, Université de Tours, Tours, France

${ }^{20}$ Service de Réanimation, Centre Hospitalier de Pau, Pau, France

${ }^{21} \mathrm{CHU}$ La Timone 2, Médecine Intensive Réanimation, Réanimation des Urgences, Aix-Marseille Université, Marseille, France

${ }^{22}$ Service de Réanimation, Centre Hospitalier Henri Mondor d'Aurillac, Aurillac,

France

${ }^{23}$ Médecine Intensive Réanimation, $\mathrm{CHU}$ de Brest, Brest, France

${ }^{24}$ Médecine Intensive Réanimation, INSERM, Université Grenoble-Alpes, U1042,

HP2, Centre Hospitalier Universitaire Grenoble Alpes, Grenoble, France

${ }^{25}$ Réanimation Médicale Archet 1, Université Cote d'Azur, CHU de Nice, Nice, France

${ }^{26}$ Service de Réanimation Médico-Chirurgicale, Centre Hospitalier de Versailles, Le

Chesnay, France

${ }^{27}$ Réanimation Médico-Chirurgicale Archet 2, INSERM U 1065, CHU de Nice, Nice, France

${ }^{28}$ Réanimation Chirurgicale, $\mathrm{CHU}$ de Poitiers, Poitiers, France

${ }^{29}$ Réanimation et USC médico-chirurgicale, CARMAS, AP-HP, Faculté de Médecine Sorbonne Université, Collegium Galilée, Hopital Tenon, Paris, France

${ }^{30}$ Service de Réanimation, Centre Hospitalier Emile Roux, Le Puy-en-Velay, France

${ }^{31}$ Réanimation Médicale et Toxicologique, AP-HP, INSERM UMR-S 942, Hopital Lariboisiere, Paris, France

Contributors J-PF and AWT (the REVA Network), in collaboration with all authors designed the study and wrote the manuscript together. SR provided substantial contributions to the conception and design of the study, and wrote the statistical analysis plan and estimated the sample size with AWT. AWT, GM, AG, RC, AD, RS, $\mathrm{FB}, \mathrm{CG}, \mathrm{LD}, \mathrm{AL}, \mathrm{SC}, \mathrm{AR}, \mathrm{EV}, \mathrm{ALM}, \mathrm{J}-\mathrm{DR}, \mathrm{KR}, \mathrm{GB}, \mathrm{CL}, \mathrm{SE}, \mathrm{WP}, \mathrm{JB}, \mathrm{GP}, \mathrm{PB}, \mathrm{NT}, \mathrm{MB}, \mathrm{GL}$, $P-E D, H N, A G, L Z, N D$ and J-PF contributed for drafting the work, revising it critically for important intellectual content and approved the final version of the manuscript. All authors gave their agreement to be accountable for all aspects of the work, and ensure the accuracy and integrity of any part of the work.

Funding The study was funded by the 'Programme Hospitalier de Recherche Clinique National 2015' of the French Ministry of Health. The study promoter is the University Hospital of Poitiers, Poitiers, France.

Disclaimer The firm Fisher \& Paykel provided the high-flow oxygen therapy equipment and masks for non-invasive ventilation to all the participating centres but has no other involvement in the study.

Competing interests AWT and J-DR report travel expenses coverage to attend scientific meetings by Fisher \& Paykel. AD and SE received research grants from Fisher \& Paykel. J-PF reports consulting fees from Fisher \& Paykel.

Patient consent Obtained.

Ethics approval The study has been approved by the central ethics committee (Ethics Committee Ouest III, Poitiers, France) with the registration number 2016A01078-43 (20 July 2016).

Provenance and peer review Not commissioned; externally peer reviewed. 
Open access This is an open access article distributed in accordance with the Creative Commons Attribution Non Commercial (CC BY-NC 4.0) license, which permits others to distribute, remix, adapt, build upon this work non-commercially, and license their derivative works on different terms, provided the original work is properly cited, appropriate credit is given, any changes made indicated, and the use is non-commercial. See: http://creativecommons.org/licenses/by-nc/4.0/.

\section{REFERENCES}

1. Thille AW, Richard JC, Brochard L. The decision to extubate in the intensive care unit. Am J Respir Crit Care Med 2013;187:1294-302.

2. Thille AW, Cortés-Puch I, Esteban A. Weaning from the ventilator and extubation in ICU. Curr Opin Crit Care 2013;19:57-64.

3. Nava S, Gregoretti C, Fanfulla F, et al. Noninvasive ventilation to prevent respiratory failure after extubation in high-risk patients. Crit Care Med 2005;33:2465-70.

4. Ornico SR, Lobo SM, Sanches HS, et al. Noninvasive ventilation immediately after extubation improves weaning outcome after acute respiratory failure: a randomized controlled trial. Crit Care 2013;17:R39.

5. Ferrer M, Valencia M, Nicolas JM, et al. Early noninvasive ventilation averts extubation failure in patients at risk: a randomized trial. $A m \mathrm{~J}$ Respir Crit Care Med 2006;173:164-70.

6. Ferrer M, Sellarés J, Valencia M, et al. Non-invasive ventilation after extubation in hypercapnic patients with chronic respiratory disorders: randomised controlled trial. Lancet 2009;374:1082-8.

7. Vargas F, Clavel M, Sanchez-Verlan P, et al. Intermittent noninvasive ventilation after extubation in patients with chronic respiratory disorders: a multicenter randomized controlled trial (VHYPER). Intensive Care Med 2017:43:1626-36.

8. Thille AW, Boissier F, Ben-Ghezala H, et al. Easily identified atrisk patients for extubation failure may benefit from noninvasive ventilation: a prospective before-after study. Crit Care 2016;20:48.

9. Rochwerg B, Brochard L, Elliott MW, et al. Official ERS/ATS clinical practice guidelines: noninvasive ventilation for acute respiratory failure. Eur Respir J 2017;50:1602426.

10. Bajaj A, Rathor P, Sehgal V, et al. Efficacy of noninvasive ventilation after planned extubation: a systematic review and meta-analysis of randomized controlled trials. Heart Lung 2015;44:150-7.

11. Peñuelas $\mathrm{O}$, Frutos-Vivar F, Fernández C, et al. Characteristics and outcomes of ventilated patients according to time to liberation from mechanical ventilation. Am J Respir Crit Care Med 2011;184:430-7.

12. Mauri T, Turrini C, Eronia N, et al. PHysiologic effects of high-flow nasal cannula in acute hypoxemic respiratory failure. Am J Respir Crit Care Med 2017;195:1207-15
13. Mauri T, Alban L, Turrini C, et al. Optimum support by high-flow nasal cannula in acute hypoxemic respiratory failure: effects of increasing flow rates. Intensive Care Med 2017;43:1453-63.

14. Delorme M, Bouchard PA, Simon M, et al. Effects of high-flow nasal cannula on the work of breathing in patients recovering from acute respiratory failure. Crit Care Med 2017;45:1981-8.

15. Maggiore SM, Idone FA, Vaschetto R, et al. Nasal high-flow versus Venturi mask oxygen therapy after extubation. Effects on oxygenation, comfort, and clinical outcome. Am J Respir Crit Care Med 2014;190:282-8.

16. Hernández G, Vaquero $C$, González P, et al. Effect of postextubation high-flow nasal cannula vs conventional oxygen therapy on reintubation in low-risk patients: A randomized clinical trial. JAMA 2016;315:1354-61

17. Hernández G, Vaquero C, Colinas L, et al. Effect of postextubation high-flow nasal cannula vs noninvasive ventilation on reintubation and postextubation respiratory failure in high-risk patients: a randomized clinical trial. JAMA 2016;316:1565-74.

18. Boles JM, Bion J, Connors A, et al. Weaning from mechanical ventilation. Eur Respir J 2007;29:1033-56.

19. Thille AW, Harrois A, Schortgen F, et al. Outcomes of extubation failure in medical intensive care unit patients. Crit Care Med 2011;39:2612-8.

20. Keenan SP, Powers C, McCormack DG, et al. Noninvasive positivepressure ventilation for postextubation respiratory distress: a randomized controlled trial. JAMA 2002;287:3238-44.

21. Esteban A, Frutos-Vivar F, Ferguson ND, et al. Noninvasive positivepressure ventilation for respiratory failure after extubation. $N$ Engl J Med 2004;350:2452-60.

22. Thille AW, Boissier F, Ben Ghezala H, et al. Risk factors for and prediction by caregivers of extubation failure in ICU patients: a prospective study. Crit Care Med 2015;43:613-20.

23. Demling RH, Read T, Lind LJ, et al. Incidence and morbidity of extubation failure in surgical intensive care patients. Crit Care Med 1988;16:573-7.

24. Epstein SK, Ciubotaru RL, Wong JB. Effect of failed extubation on the outcome of mechanical ventilation. Chest 1997;112:186-92.

25. Girault C, Bubenheim M, Abroug F, et al. Noninvasive ventilation and weaning in patients with chronic hypercapnic respiratory failure: a randomized multicenter trial. Am J Respir Crit Care Med 2011;184:672-9.

26. Khilnani GC, Galle AD, Hadda V, et al. Non-invasive ventilation after extubation in patients with chronic obstructive airways disease: a randomised controlled trial. Anaesth Intensive Care 2011;39:217-23.

27. Su CL, Chiang LL, Yang SH, et al. Preventive use of noninvasive ventilation after extubation: a prospective, multicenter randomized controlled trial. Respir Care 2012;57:204-10.

28. Vargas F, Saint-Leger M, Boyer A, et al. Physiologic Effects of HighFlow Nasal Cannula Oxygen in Critical Care Subjects. Respir Care 2015;60:1369-76. 